PACS 79.20.Fv

\title{
Features of Auger-emission in channeling
}

\author{
I.A. Kossko, A.Ye. Denisov ${ }^{1}$ \\ Institute for Problems of Materials Science, 3, vul. Krzhizhanovskogo, 03142 Kyiv, Ukraine \\ Kossko@ipms.kiev.ua \\ ${ }^{l}$ Center for Nanotechnologies RT, 50, ul. Peterburgskaya, 420107 Kazan, Russia \\ Alexey.Denisov@nanort.ru
}

\begin{abstract}
Shown in this paper is the influence of channeling effect on formation of the signal for low- and high-energy Auger-electrons observed in monocrystalline silicon. It has been ascertained the anisotropic (wave-like) character of the yield value for lowenergy Auger-electrons in silicon, when changing the angle of acting initial radiation during sample rotation.
\end{abstract}

Keywords: Auger-electron, channeling, angular anisotropy.

Manuscript received 10.10.14; revised version received 20.02.14; accepted for publication 20.03.14; published online 31.03.14.

\section{Introduction}

In the process of electron- and ion-probe investigations of monocrystalline materials, it is necessary to take into account some artefacts of analysis, which are related with channeling effects inherent to electrons and ions. Account of these artefacts in analysis enables to enhance reliability of quantitative analysis, to give correct interpretation of mapping the surface in characteristic emission of various components in single crystals and thin monocrystalline films that are used in scienceintensive branches of up-to-date materials science.

Performed in the works $[1,2]$ are investigations of anisotropy inherent to characteristic X-ray radiation and Auger-electrons as well as for secondary electron emission from single crystals at mean energies of electrons (diffraction and localization of electrons in crystals).

In the process of axial channeling, when a particle moves between adjaicent rows of atoms, the yield value for Auger-electrons has its maximum. The trajectory of particle channeling is located further from atomic nuclei than that of non-channeling particles. It results in enhancing the free path of particles in this channel as compared with the case when channeling is absent. It is a right consequence of the fact that the electron density in channels is lower than the averaged one inside the crystal. Since the channeling particles move comparatively far from nuclei and their electron shells, the probability to transfer energy to electrons related with lattice atoms as well as the probability of intrinsic processes are considerably lower. When interacting with impurity elements or structural defects at the surface of single crystals, the channeling effect is lowered by two orders $[3-6]$.

This methodical work is devoted to analysis of anisotropy inherent to characteristic emission of a definite chemical element possessing low- and highenergy Auger-electrons in channeling conditions.

A different influence of the channeling process on the yield value of low- and high-energy Auger-electrons has obviously a general character. In this work, as a reference sample we used monocrystalline silicon (100) with a layer of its oxide on the surface, which was formed due to contact with ambient atmosphere. When using the ionic etching for $3 \mathrm{~min}$, the oxide layer was entirely removed, and the channeling effect of electrons in monocrystalline silicon became visible. In the process of channeling, the yield of low- and high-energy Augerelectrons of silicon acquired a selective character. 
Fig. 1 shows schematically the experimental setup. We used the Auger-spectrometer JAMP-9500 (JEOL), $U_{a c c}=10 \mathrm{kV}, I_{a b s}=5 \cdot 10^{-9} \mathrm{~A}$, and the diameter of the acting electron beam $D=2 \mu \mathrm{m}$. Ion bombardment (depth profiling) was realized using argon ions accelerated with the potential difference $\mathrm{U}_{\mathrm{acc}}=3 \mathrm{kV}$. During the process of profiling, we used continuous rotation of the sample with a constant velocity. The angle between the normal to the sample surface and acting electron probe was $60^{\circ}$. The period of oscillations in the flow of low-energy Auger-electrons corresponded to sample rotation by $360^{\circ}$.

Shown in Fig. 2 is the scheme of interaction between the electron probe and atomic planes, where $\mathbf{A}$ corresponds to the enhanced yield of low-energy electrons, while $\mathbf{B}-$ to the lowered yield of low-energy electrons, which is caused by screening related with the neighboring atomic layer.

Represented in Fig. 3 are ranges of energies (the so-called "windows") for registration of the yields of low- and high-energy Auger-electrons of silicon and oxygen. The middle part of the figure shows the energy range of oxygen. Absence of any oxygen peak corresponds to full etching out it by argon ions. The top part of this figure shows its integrated form, while the bottom one - differential form used for registration of Auger signals.

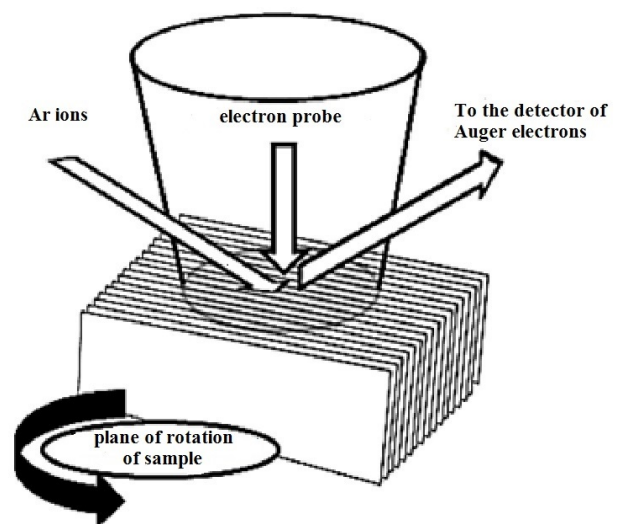

Fig. 1. Experimental scheme.

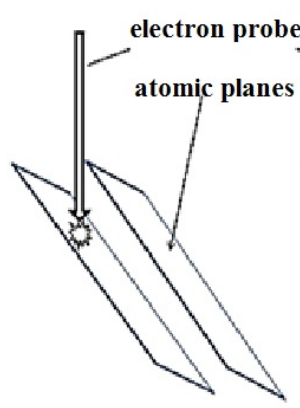

A

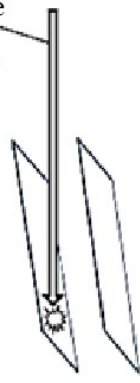

B
Fig. 2. Scheme of interaction between the electron probe and atomic planes. The cases of enhanced (A) and lowered (B) yield of low-energy electrons.

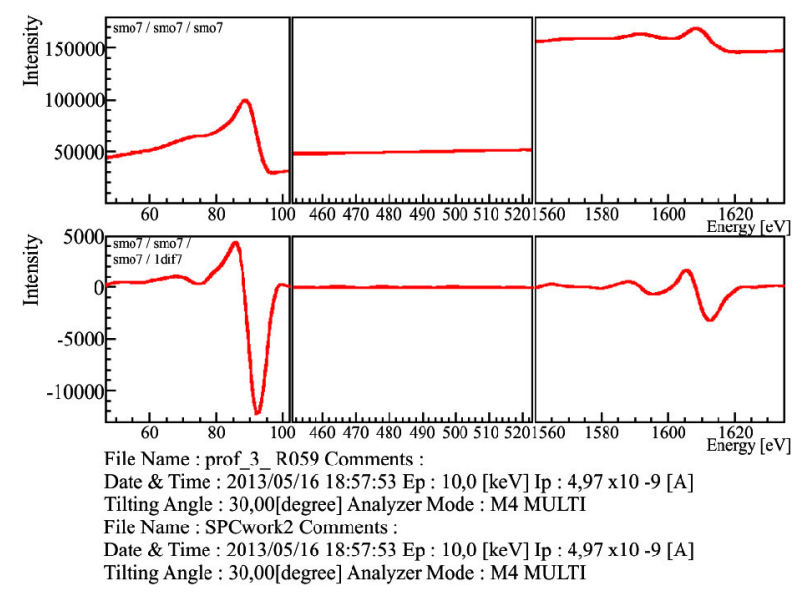

Fig. 3. Energy ranges of Auger spectra inherent to $\mathrm{Si}_{L V V}$ (left part), $\mathrm{Si}_{K L L}$ (right part), $\mathrm{O}_{K L L}$ (middle part).

Shown in Figs 4 and 5 are temporal dependences for the Auger-electron yield of $\mathrm{Si}_{L V V}$ (a), $\mathrm{O}_{K L L}$ (b), $\mathrm{Si}_{K L L}(\mathrm{c})$ in the cases of ion bombardment with simultaneous rotation of the sample in 3D and 2D forms, respectively. The oscillation process in the yield of $\mathrm{Si}_{L V V}$ Auger-electrons takes place in $4 \mathrm{~min}$ of the ion etching after full removing the oxide layer and stabilization of the channeling process under the variable rotation angle between the incident electron beam and crystal planes. Channeling the electrons, that is movement of particles along the "channels" between parallel rows of atoms (planes) has a different probability dependent on the enter angle of the electron beam into the lattice.

So, if an electron trajectory is directed along atomic planes (plane channeling), then these electrons are subjected to only sliding collisions with a practically unchanged momentum, which results in a lowered probability of vacancy creation at the low-lying energy levels. In its turn, it leads to a decreased yield of Augerelectrons.

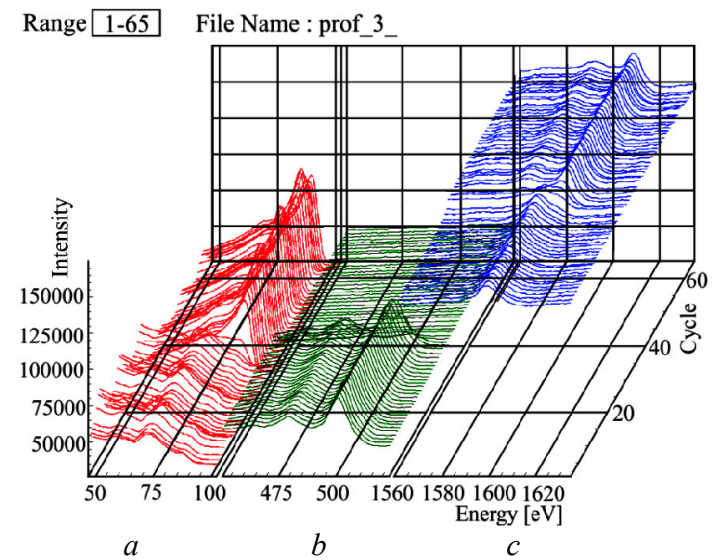

Fig. 4. Temporal dependence of the Auger-electron yield from $\mathrm{Si}_{L V V}$ (a), $\mathrm{O}_{K L L}$ (b), $\mathrm{Si}_{K L L}$ (c) for the case of ion bombardment with simultaneous rotation of the sample. 


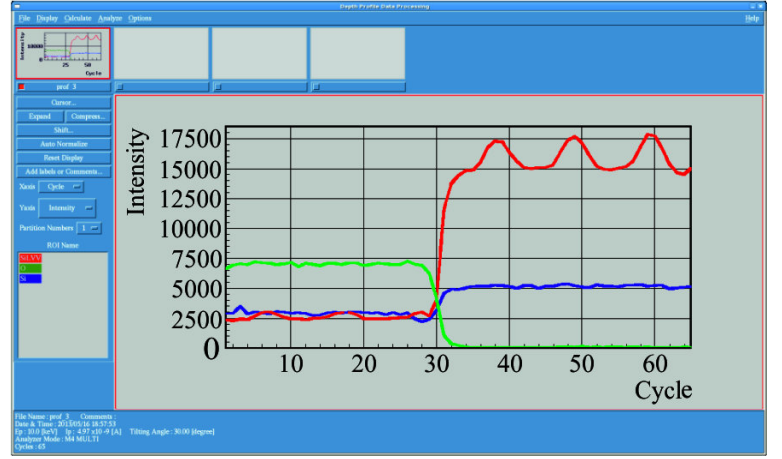

Fig. 5. 2D form of presentation for the depth distribution of silicon and oxyhen with simultaneous rotation of the sample: $\mathrm{Si}_{L V V}(\mathrm{a}), \mathrm{Si}_{K L L}(\mathrm{~b}), \mathrm{O}_{K L L}(\mathrm{c}), \mathrm{a}-\mathrm{red}, \mathrm{b}$ - blue, $\mathrm{c}$ - green (in the electronic version of the paper).

In this work, the channeling effect was realized in various forms. It depended on the angle of incidence for the initial electron beam on the surface of monocrystalline silicon. Practical constancy of the signal level for $\mathrm{Si}_{K L L}$ (b) state in Fig. 5 is indicative of the fact that the degree of screening for high-energy electrons is insignificant.

Contribution of silicon Auger-electrons to emission due to ion bombardment for LVV and KLL transitions also has a selective character, however, the fact that measurements of the emission yield take place out of the etching process allows not to take this contribution into account.

\section{References}

1. V.V. Balashov, A.A. Sokolik, A.V. Stysin. Angular anisotropy of characteristic X-ray radiation and Auger-electrons in the process of resonant coherent excitation of relativistic ions during their plane channeling // Zhurnal eksperimental'noi $i$ teoreticheskoi fiziki, 134(1), p. 164 - 171 (2008), in Russian.

2. M.V. Gomoyunova. Anisotropy of secondary electron emission from single crystals of transition metals for mean electron energies (diffraction and localization of electrons in crystals). Doctoral thesis (Phys\&Math Sciences) by the speciality 01.01.04 - Physical electronics, including the quantum one. Leningrad, 1984, 388 pages, in Russian.

3. A.F. Tulikov. Influence of crystal lattice on some atomic and nuclear processes // Uspekhi fizicheskikh nauk, 87(4), p. 585 (1965), in Russian.

4. I. Lindhard. Influence of crystal lattice on movement of fast charged particles// Uspekhi fizicheskikh nauk, 99(2), p. 249 (1969), in Russian.

5. M. Tompson. Channeling particles in crystals // Uspekhi fizicheskikh nauk, 99(2), p. 297 (1969), in Russian.

6. Yu.M. Kagan, Yu.V. Kononets. Theory of channeling effect // Zhurnal eksperimental'noi $i$ teoreticheskoi fiziki, 58(1), p. 226 (1970), in Russian. 